\title{
Geographic pattern of central retinal sensitivity after intravitreal triamcinolone for diabetic macular edema
}

\author{
Kazuaki Nishijima
}

Received: 11 March 2011 /Accepted: 14 March 2011 /Published online: 6 April 2011

(C) Springer-Verlag 2011

\section{Dear Editor,}

We thank Gundogan et al. for their comments and appreciate the opportunity to respond. Our data showed that visual acuity (VA) and central macular thickness improved significantly at 1 week and 1 month after intravitreal injection of triamcinolone acetonide (IVTA), which was the same result as shown in many previous reports. In our study, mean retinal sensitivity evaluated by microperimeter (MP-1; Nidek Technologies, Padua, Italy), however, showed no significant improvement at 1 week, but did show significant improvement at 1 month. Mean retinal sensitivity of the points at the central $2^{\circ}$, which was significantly lower than that at 6 and $10^{\circ}$ before treatment, showed improvement similar to those of 6 and $10^{\circ}$ after IVTA. These data suggest that IVTA for diabetic macular edema (DME) showed significant functional recovery of macular area to a certain degree with a time lag between the morphologic and the functional recovery in DME.

According to the suggestion of Dr. Gundogan, we reviewed the medical records of all the cases in this study to collect data about the duration of diabetes and DME. After all, it was impossible to complete it. The onset of diabetes/DME was unclear from our medical records in some patients. This is a limitation of the current study because of its retrospective nature.

Today, some reports have demonstrated that the status of photoreceptor inner and outer segments (IS/OS) line in macular area on OCT images had a correlation with visual function in several diseases. [1-6] In the present study, VA was significantly better in the IS/OS(+) group than in the IS/ OS(-) group at 1 month postoperatively. In addition, retinal

\section{K. Nishijima $(\triangle)$}

Department of Ophthalmology,

Kyoto University Graduate School of Medicine,

54 Shogoin Kawahara-cho, Sakyo-ku, Kyoto 606-8507, Japan

e-mail: nissi@kuhp.kyoto-u.ac.jp sensitivity at $2^{\circ}$, which correspond to $500 \mu \mathrm{m}$ from the center of the fovea, was also better in the IS/OS(+) group than in the IS/OS(-) group. Time-domain OCT cannot visualize the status of IS/OS in the presence of DME, whereas the latest spectral-domain OCT can detect the changes of outer retinal layer even in the presence of DME. This new instrument would enable us to evaluate intrinsic neurophysiological changes and to predict the clinical course of DME after treatment by evaluating the outer retinal status.

Conflict of interest No conflicts of interest or commercial or proprietary interests exist for any of the authors.

\section{References}

1. Oishi A, Otani A, Sasahara M, Kojima H, Nakamura H, Kurimoto M, Yoshimura N (2009) Photoreceptor integrity and visual acuity in cystoid macular oedema associated with retinitis pigmentosa. Eye 23:1411-1416

2. Spaide RF, Koizumi H, Freund KB (2008) Photoreceptor outer segment abnormalities as a cause of blind spot enlargement in acute zonal occult outer retinopathy-complex diseases. Am J Ophthalmol 46:111-120

3. Ojima Y, Tsujikawa A, Hangai M, Nakanishi H, Inoue R, Sakamoto A, Yoshimura N (2008) Retinal sensitivity measured with the micro perimeter 1 after resolution of central serous chorioretinopathy. Am J Ophthalmol 146:77-84

4. Ota M, Tsujikawa A, Murakami T, Yamaike N, Sakamoto A, Kotera Y, Miyamoto K, Kita M, Yoshimura N (2008) Foveal photoreceptor layer in eyes with persistent cystoid macular edema associated with branch retinal vein occlusion. Am J Ophthalmol 145:273-280

5. Unoki N, Nishijima K, Kita M, Hayashi R, Yoshimura N (2010) Structural changes of fovea during remission of Behçet's disease as imaged by spectral domain optical coherence tomography. Eye 24:969-975

6. Sakamoto A, Nishijima K, Kita M, Oh H, Tsujikawa A, Yoshimura N (2009) Association between foveal photoreceptor status and visual acuity after resolution of diabetic macular edema by pars plana vitrectomy. Graefes Arch Clin Exp Ophthalmol 247:1325-1330 